\section{Do Thiols Merely Passivate Gold Nanoclusters?}

Recently, Häkkinen, Barnett, and Landman (HBL) [1] investigated the properties of a thiol-passivated gold cluster, using density functional theory (DFT). They considered a truncated-octahedral face-centered-cubic (TO-fcc) structure for the $\mathrm{Au}_{38}$ cluster, coated with a monolayer of 24 methylthiol $\left(\mathrm{SCH}_{3}\right)$ molecules. After a structural relaxation, they found: (i) that the octahedral symmetry persisted in the relaxed structure; (ii) small inward and outward relaxations of the different gold atoms; (iii) a charge transfer of $2 e$ at the well-defined gold-thiol interface.

Here, we point out that the thiol monolayer has a much stronger effect than that reported by HBL [1]. We have used DFT, scalar-relativistic norm-conserving pseudopotentials, and a double- $\zeta$ basis set of numerical atomic orbitals [2]. Other details are similar to those used in our studies of bare gold clusters [3,4]. We have performed conjugate-gradient relaxations starting from different cluster-monolayer configurations. These include the one proposed by HBL and the same configuration with random atomic displacements to break the symmetry. Unless the octahedral symmetry is assumed, we invariably find a dramatic distortion (relaxed coordinates are available upon request). The final, stable structure is $\approx 4 \mathrm{eV}$ lower in energy than that of the TO-fcc geometry. This energy difference has been reproduced using an independent, standard plane-wave (PW) pseudopotential code [5] and the same pseudopotential parameters and PW cutoff as HBL. In the relaxed structure, the inner cuboctahedron deforms into a disordered cluster core (a central atom surrounded by 15 atoms) made of distorted pentagonal bipyramid subunits. The ill-defined gold-thiol interface is formed by the remaining 22 gold atoms sitting at irregular

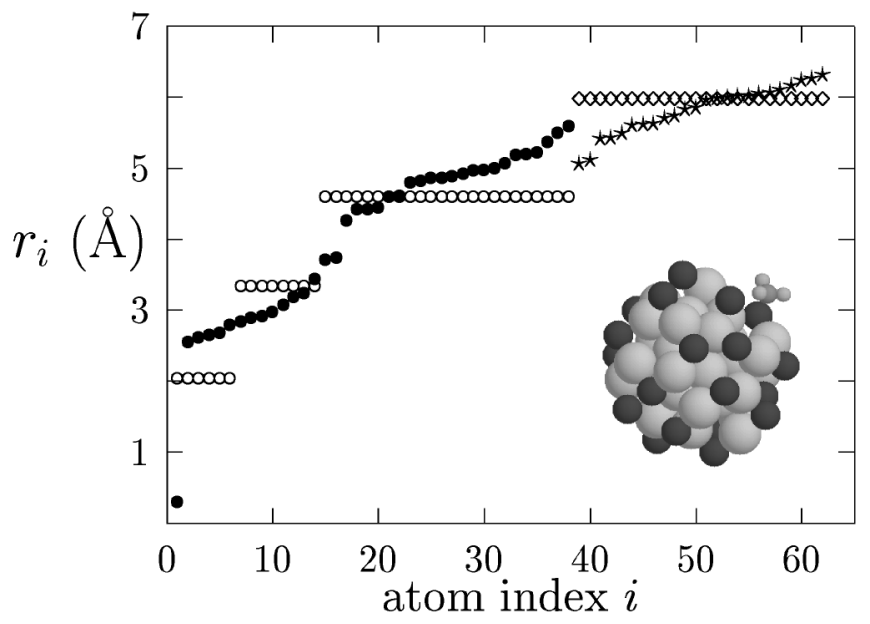

FIG. 1. Distances of the gold $(i=1-38)$ and sulfur $(i=39-62)$ atoms from the cluster center of mass for the $\mathrm{Au}_{38}\left(\mathrm{SCH}_{3}\right)_{24}$ nanocluster in the disordered (closed circles and stars) and TO-fcc (open circles and diamonds) structures. The inset shows the relaxed disordered structure. Sulfur atoms are depicted as darker spheres and only one $\mathrm{CH}_{3}$ group is shown. positions, and by the sulfur atoms of the thiol heads incorporated into the cluster surface. Figure 1 shows the clearly separated gold and sulfur atomic shells of the TO-fcc structure proposed by HBL and their distortion and overlap in our disordered configuration. Different patterns of charge transfer occur in this disordered, alloyed cluster surface, in contrast with the uniform charge transfer at the well-defined interface found by HBL [1].

Although the physicochemical details of the goldthiol interaction in nanoparticle-size systems remain to be investigated, the existence of stable disordered gold nanoclusters is not unexpected. Evidence from several theoretical studies [3,4,6-8] (consistent with experiments, see Refs. $[7,8])$ shows that the lowest-energy structures of bare $\mathrm{Au}_{38}, \mathrm{Au}_{55}$, and $\mathrm{Au}_{75}$ nanoclusters are disordered.

In conclusion, we find that a methylthiol monolayer produces a dramatically distorted $\mathrm{Au}_{38}$ nanocluster with a disordered Au-S alloy interface. This is in sharp contrast to the claim of HBL [1], that its effect reduces to a radial relaxation of the TO-fcc geometry. We assign this discrepancy to an insufficient sampling of configuration space by HBL.

This work was supported by Mexico's CONACYT Grants No. 25083-E and No. 28822-E, and DGAPAUNAM Project No. IN101297. I. L. G. and J. M. S. thank Spain's SEUID for financial support.

\section{L. Garzón, ${ }^{1,2}$ C. Rovira ${ }^{3}$ K. Michaelian, ${ }^{2}$ M. R.}

Beltrán, ${ }^{4}$ P. Ordejón, ${ }^{5}$ J. Junquera, ${ }^{1}$ D. Sánchez-Portal, ${ }^{1,6}$ E. Artacho, ${ }^{1}$ and J. M. Soler ${ }^{1,7}$

${ }^{1}$ Departamento de Física de la Materia Condensada C-III
Universidad Autónoma de Madrid
E-28049 Madrid, Spain
${ }^{2}$ Instituto de Física
Universidad Nacional Autónoma de México
Apartado Postal 20-364
México D.F., 01000 México
${ }^{3}$ Universitat de Barcelona
Marti i Franques 1
08028 Barcelona, Spain
${ }^{4}$ Instituto de Investigaciones en Materiales, UNAM
Apartado Postal 70-360
México D.F., 01000 México
${ }^{5}$ Institut de Ciéncia de Materials de Barcelona-CSIC
08193 Bellaterra
Barcelona, Spain
${ }^{6}$ Department of Physics and Materials Research Laboratory
University of Illinois
Urbana, Illinois 61801
${ }^{7}$ Department of Physics, Lyman Laboratory
Harvard University
Cambridge, Massachusetts 02138

Received 30 March 2000; revised manuscript received 26 June 2000

PACS numbers: $36.40 . \mathrm{Cg}, 36.40 . \mathrm{Mr}, 61.46 .+\mathrm{w}$

[1] H. Häkkinen et al., Phys. Rev. Lett. 82, 3264 (1999). 
[2] E. Artacho et al., Phys. Status Solidi B 215, 809 (1999), and references therein.

[3] I. L. Garzón et al., Phys. Rev. Lett. 81, 1600 (1998).

[4] J. M. Soler et al., Phys. Rev. B 61, 5771 (2000).

[5] J. Hütter, H. P. Luthi, and M. Parrinello, Comput. Mater. Sci. 2, 244 (1994); CPMD program version 3.0, written by J. Hütter, Max-Planck-Institut für Festkörperforschung, Stuttgart, 1996.

[6] I. L. Garzón et al., Phys. Rev. B 54, 11796 (1996).

[7] K. Michaelian et al., Phys. Rev. B 60, 2000 (1999).

[8] I. L. Garzón et al., Eur. Phys. J. D 9, 211 (1999). 